\title{
Does perineural invasion in a radical prostatectomy specimen predict biochemical recurrence in men with prostate cancer?
}

\author{
Fairleigh Reeves, MBBS; ${ }^{*}$ Christopher M. Hovens, PhD; ${ }^{*+}$ Laurence Harewood, BSc, MBBS, FRACS; ${ }^{*+}$ \\ Shane Battye, MBBS, BMedSc, FRCPA; ; Justin S. Peters, MBBS, FRACS; ${ }^{*+}$ \\ Anthony J. Costello, MBBS, MD, FRACS, FRCSI (Hon); Niall M. Corcoran, MBBCh, BAO, PhD, FRACS ${ }^{* t}$
}

*Department of Urology and Surgery, University of Melbourne, The Royal Melbourne Hospital, Australia; ‘'Australian Prostate Cancer Research Centre Epworth, Richmond, Australia; $\$$ TissuPath Pty Ltd, Mount Waverley, Australia

Cite as: Can Urol Assoc J 2015;9(5-6):E252-5. http://dx.doi.org/10.5489/cuaj.2619

Published online May 13, 2015.

\section{Abstract}

Introduction: The ability of perineural invasion ( $\mathrm{PNI}$ ) in radical prostatectomy (RP) specimens to predict biochemical recurrence $(B C R)$ is unclear. This study investigates this controversial question in a large cohort.

Methods: A retrospective analysis was undertaken of prospectively collected data from 1497 men who underwent RP (no neoadjuvant therapy) for clinically localized prostate cancer. The association of $\mathrm{PNI}$ at RP with other clinicopathological parameters was evaluated. The correlation of clinicopathological factors and BCR (defined as prostate-specific antigen [PSA] $>0.2 \mathrm{ng} / \mathrm{mL}$ ) was investigated with univariable and multivariable Cox regression analysis in 1159 men. Results: PNI-positive patients were significantly more likely to have a higher RP Gleason score, pT3 disease, positive surgical margins, and greater cancer volume $(p<0.0005)$. The presence of PNI significantly correlated with BCR on univariable (hazard ratio 2.30, $95 \%$ confidence interval $1.50-3.55, p<0.0005)$, but not multivariable analysis $(p=0.602)$. On multivariable Cox regression analysis the only independent prognostic factors were preoperative PSA, RP Gleason score, pT-stage, and positive surgical margin status. These findings are limited by a relatively short follow-up time and retrospective study design.

Conclusions: $\mathrm{PNI}$ at RP is not an independent predictor of BCR. Therefore, routine reporting of $\mathrm{PNI}$ is not indicated. Future research should be targeted at the biology of PNI to increase the understanding of its role in prostate cancer progression.

\section{Introduction}

One of the greatest challenges in the management of prostate cancer is distinguishing aggressive from indolent disease, thus determining which patients will relapse and require further management.

Perineural invasion (PNI) is considered a major mechanism of extraprostatic spread of prostate cancer. ${ }^{1}$ It is evident on histological examination of radical prostatectomy (RP) specimens in a significant proportion of cases. ${ }^{2,3}$ Theoretically the presence of $\mathrm{PNI}$ on RP specimens may correlate with risk of disease progression. However, the prognostic significance of this is controversial and evidence in this area has been derived from relatively small cohort studies.

The aim of this study was to evaluate the ability of PNI to predict biochemical recurrence (BCR) in a large cohort of men with clinically localized prostate cancer and to determine if routine pathological reporting is justified.

\section{Methods}

Consecutive patients with localized prostate cancer, treated with RP between December 2005 and October 2012 were included in this study. Participants were retrospectively identified from our ethics approved institutional prostate cancer database. Those with no PNI data recorded and those who received neoadjuvant hormonal therapy were excluded.

Our prostate cancer database prospectively collects clinical and pathological data for patients undergoing RP.

The surgical specimen surface was inked, and the specimen was formalin fixed, paraffin embedded and processed in its entirety. The seminal vesicles were truncated at their base. After a 5 to $10-\mathrm{mm}$ segment from the apical margin, the prostate was transversely sectioned perpendicular to the urethra at 4-mm intervals. Serial sections of the slices from the apical margins and the base were cut radially. Fivemicrometre sections were stained with hematoxylin and eosin. The histological analysis and reporting of our RP specimens were performed by expert uropathologists. PNI status recorded in our database was extracted from routine clinical RP histology reports and was defined as prostate cancer within the perineural space. It did not differentiate between intra- and extraprostatic $\mathrm{PNI}$, nor was there quantification of the extent of PNI.

Serum prostate-specific antigen (PSA) was measured 3, 6 , and 12 months, then yearly thereafter. Follow-up was 
censored at the time of BCR, which for the purpose of this study was defined as a single PSA reading of $\geq 0.2 \mathrm{ng} / \mathrm{mL}$ or the commencement of salvage therapy (where treatment occurred prior to PSA reaching $0.2 \mathrm{ng} / \mathrm{mL}$ ).

The relationships between $\mathrm{PNI}$ and other clinicopathological factors were evaluated using Chi square statistic for categorical variables, and Mann-Whitney $U$ test for nonparametric continuous variables. Kolmogorov-Smirnov and Shapiro-Wilk tests were used to verify the distribution of continuous variables (i.e., PSA and cancer volume). KaplanMeier curves (with log-rank testing for significance) were used for univariable analysis of the relationship between clinicopathological variables (including PNI) and BCR-free survival. Multivariable Cox regression analysis was performed to identify independent predictors of BCR-free survival. All statistical analyses were performed using SPSS Statistics v17.0.

\section{Results}

\section{Patient characteristics}

In total, 1497 patients were identified during the study period meeting our inclusion criteria. The median age of participants was 62. Most had pathological T2 stage (69.7\%) disease and Gleason score 7 (77.8\%). PNI was identified in the specimens of $1173(78 \%)$ participants (Table 1).

Follow-up data were available for 1159 patients, of these $238(20.5 \%)$ had PSA recurrence. The median time to recurrence was 8 months after RP and the median follow-up for patients who did not recur was 15 months.

\section{Relationship between PNI and other clinicopathological parameters}

Chi square testing demonstrated that PNI positive patients were significantly more likely to have a higher RP Gleason

\begin{tabular}{|c|c|c|}
\hline \multicolumn{2}{|l|}{ Baseline characteristics ( $n=1497$ ) } & Median (IQR) \\
\hline \multicolumn{2}{|l|}{ Age (at operation) } & $62(8.79)$ \\
\hline \multirow{4}{*}{ Preoperative PSA } & & $6.3(3.8)$ \\
\hline & $<10(\%)$ & $1240(82.8)$ \\
\hline & 10-20 (\%) & $197(13.2)$ \\
\hline & $>20(\%)$ & $60(4)$ \\
\hline \multirow{3}{*}{ Clinical T-stage } & $1(\%)$ & 1009 (67.4) \\
\hline & $2(\%)$ & $448(29.9)$ \\
\hline & $3(\%)$ & $37(2.5)$ \\
\hline \multicolumn{3}{|l|}{ RP pathology characteristics $(n=1497$ ) } \\
\hline \multirow{3}{*}{ Gleason score } & $\leq 6$ & $191(12.8)$ \\
\hline & $=7$ & $1164(77.8)$ \\
\hline & $\geq 8$ & $142(9.5)$ \\
\hline \multirow{2}{*}{ pT-stage } & $\mathrm{T} 2$ & $1042(69.7)$ \\
\hline & T3 & $454(30.3)$ \\
\hline Perineural invasion (\%) & & $1173(78.4)$ \\
\hline Extraprostatic extension (\%) & & $448(29.9)$ \\
\hline Seminal vesicle invasion (\%) & & $92(6.1)$ \\
\hline Positive surgical margin (\%) & & $390(26.1)$ \\
\hline Cancer volume (cc), Median (IQR) & & $2.1(3.2)$ \\
\hline \multicolumn{3}{|l|}{ Follow-up ( $n=1159$ ) } \\
\hline \multicolumn{2}{|l|}{ PSA recurrence } & $238(20.5)$ \\
\hline \multicolumn{2}{|c|}{ Median (IQR) time to recurrence (months) } & $8(22)$ \\
\hline \multirow{2}{*}{ Median (IQR) follow up (months) } & Overall & $14(24)$ \\
\hline & No recurrence & $15(24)$ \\
\hline
\end{tabular}

score, pT3 disease and the presence of positive surgical margin. Similarly, patients with PNI had significantly greater cancer volume on Mann-Whitney $U$ testing. No significant relationship was seen between age or preoperative PSA and PNI (Table 2).

\begin{tabular}{|c|c|c|c|c|c|}
\hline Variable & & $\mathbf{N}(\%)$ & PNI present (\%) & PNI absent (\%) & $p$ value \\
\hline \multirow{2}{*}{ Age } & & Median & 62.05 & 61.84 & \multirow{2}{*}{0.341} \\
\hline & & IQR & 8.94 & 8.05 & \\
\hline \multirow{2}{*}{\multicolumn{2}{|c|}{ Preoperative PSA }} & Median & 6.35 & 6.1 & \multirow{2}{*}{0.225} \\
\hline & & IQR & 3.9 & 3.9 & \\
\hline \multirow{2}{*}{ Cancer volume } & & Median & 2.60 & 0.70 & \multirow{2}{*}{$<0.0005$} \\
\hline & & IQR & 3.3 & 1.7 & \\
\hline \multirow{3}{*}{ RP Gleason score } & $\leq 6$ & $191(12.8)$ & $70(36.6)$ & $121(63.4)$ & \multirow{3}{*}{$<0.0005$} \\
\hline & $=7$ & 1164 (77.8) & 972 (83.5) & $192(16.5)$ & \\
\hline & $\geq 8$ & $142(9.5)$ & $131(92.3)$ & $11(7.7)$ & \\
\hline \multirow{2}{*}{ pT-stage } & $\mathrm{T} 2$ & $1042(69.7)$ & $746(71.6)$ & $296(28.4)$ & \multirow{2}{*}{$<0.0005$} \\
\hline & T3 & $454(30.3)$ & $426(93.8)$ & $28(6.2)$ & \\
\hline \multirow{2}{*}{ Positive surgical margin } & - & 1105 (73.9) & $834(75.5)$ & $271(24.5)$ & \multirow{2}{*}{$<0.0005$} \\
\hline & + & $390(26.1)$ & $337(86.4)$ & $53(13.6)$ & \\
\hline
\end{tabular}

PNI: perineural invasion; PSA: prostate-specific antigen; RP: radical prostatectomy; IQR: interquartile range. 
Reeves et al.

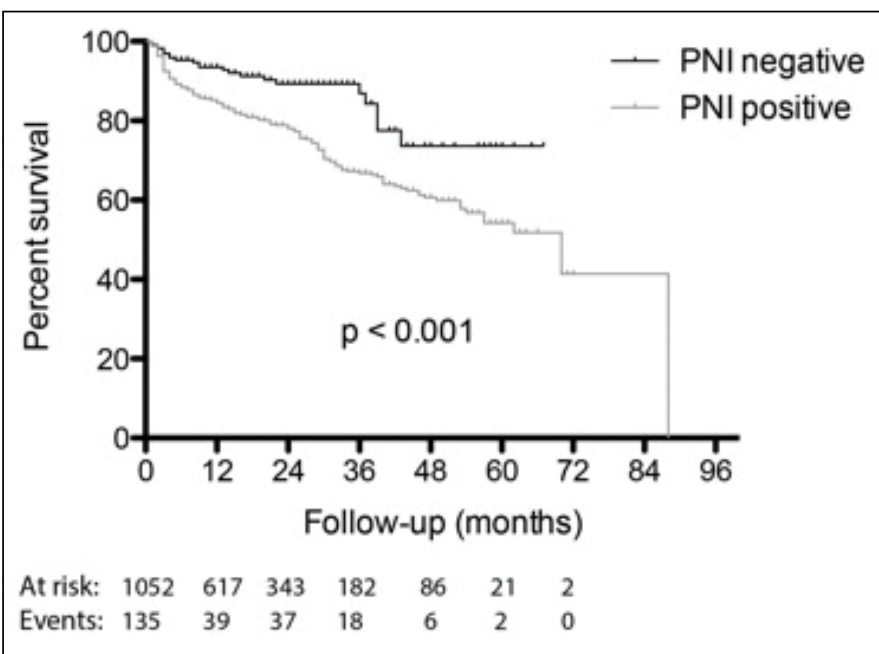

Fig. 1. Probability of biochemical recurrence-free survival stratified by perineural invasion status on univariable analysis.

\section{Predictors of biochemical recurrence}

All clinicopathological variables tested on univariable analysis, except age, were significantly associated with BCR (Fig. 1, Table 3). Of the PNI-negative patients, 9.7\% had a BCR, compared to $23.3 \%$ of PNI-positive patients (hazard ratio $2.30,95 \%$ confidence interval $1.50-3.55, p<0.0005$ ). On multivariable Cox regression analysis, PNI failed to retain significance (Table 3). Variables shown to be independent predictors of BCR were preoperative PSA, cancer volume, RP Gleason score, pT-stage, and surgical margin status.

\section{Discussion}

Prediction of BCR in prostate cancer remains imprecise. More sensitive and specific markers of risk are needed. Many institutions routinely report PNI despite the uncertainty of its prognostic significance.

This study has clearly demonstrated that PNI is a common finding in RP specimens that does not provide any additional information for BCR risk prediction, beyond that of routine clinicopathological data. In this cohort, the presence of PNI was significantly related to RP Gleason score, pT-stage, positive surgical margin status, and cancer volume. In light of the close relationship between $\mathrm{PNI}$ and variables known to predict $\mathrm{BCR}$, it is not surprising that many studies (including this one) have demonstrated that $\mathrm{PNI}$ is associated with BCR on univariable analysis. However, PNI was not shown to be an independent predictor of BCR in this cohort.

One of the strengths of this study is its size. It is the largest study we are aware of that reports the ability of PNI (in RP) to predict BCR. Potential limitations of this study are its retrospective technique and its relatively short follow-up time (median 15 months). We recognize that the median follow-up was short, which reflected the tertiary nature of our practice, as well as shared care and/or early discharge of patients with their primary physician. Despite this however, over $20 \%$ of patients in the cohort experienced BCR. As histology reports were generated as a part of routine clinical care, PNI was simply reported as present or absent; no quantification of PNI was provided. However, because this reflects the real clinical setting, results should be applicable to everyday practice.

As the pathogenesis of $\mathrm{PNI}$ is not well-understood, it is difficult to explain the findings of clinical studies in this area. $\mathrm{PNI}$ is a route of metastasis for many different types of cancer (particularly pancreatic, but also including head and neck, bladder, biliary and colorectal cancers) ${ }^{4}$ It was previously thought to be due to direct lymphatic spread into nerves, but this has subsequently been challenged, as lymphatic channels do not appear to penetrate the epineurium. Later, the nerve sheath was presumed to provide a low-resistance path for tumour spread. ${ }^{4,5}$ Most recently it has been discovered that the process of $\mathrm{PNI}$ is not passive, but instead PNI occurs as a result of symbiotic interactions between nerves and malignant cells that result in co-stimulation of growth. The nerve microenvironment affords prostate cancer cells a survival advantage that is evident through reduced apoptotic activity and increased proliferation compared to prostate

\begin{tabular}{|c|c|c|c|c|}
\hline \multirow[t]{2}{*}{ Covariates } & \multirow{2}{*}{$\begin{array}{c}\text { Univariable survival analysis } \\
\text { p value }\end{array}$} & \multicolumn{3}{|c|}{ Multivariable Cox regression analysis } \\
\hline & & HR & $95 \% \mathrm{Cl}$ & $p$ value \\
\hline Age & 0.203 & 1.00 & $0.98-1.02$ & 0.990 \\
\hline Preoperative PSA & $<0.0005$ & 1.02 & $1.01-1.03$ & 0.002 \\
\hline Cancer volume & $<0.0005$ & 1.03 & $1.00-1.06$ & 0.034 \\
\hline \multicolumn{5}{|l|}{ RP Gleason score: } \\
\hline$\leq 6$ vs 7 & 0.003 & 1.55 & $0.84-2.87$ & 0.164 \\
\hline$\leq 6$ vs $\geq 8$ & $<0.0005$ & 3.27 & $1.64-6.52$ & 0.001 \\
\hline pT-stage (pT2, pT3) & $<0.0005$ & 1.96 & $1.44-2.66$ & $<0.0005$ \\
\hline Perineural invasion & $<0.0005$ & 1.14 & $0.71-1.83$ & 0.602 \\
\hline Positive surgical margin & $<0.0005$ & 1.88 & $1.40-2.50$ & $<0.0005$ \\
\hline
\end{tabular}


cancer cells in non-PNI sites. ${ }^{6}$ Further basic science research is needed to elucidate the biology of PNI to inform targeted clinical research.

Our findings are consistent with most published literature in this area. Although a number of studies have found that $\mathrm{PNI}$ predicts $\mathrm{BCR}$ on univariable analysis, ${ }^{7-15}$ only two studies have reported $\mathrm{PNI}$ as a significant predictor on multivariable analysis. ${ }^{8,13}$ Both of these studies examined relatively small cohorts of fewer than 250 participants. Contrary to most studies, Ramsden and colleagues ${ }^{16}$ reported that the presence of PNI predicted improved clinical outcome, which as the authors acknowledged, is counterintuitive.

In our cohort PNI was identified in $78 \%$ of all patients. Previously reported rates of $\mathrm{PNI}$ in RP specimens vary between $32 \%$ and $99 \% .^{2,13}$ Differences in PNI rates may be attributable to dissimilar disease severity in study cohorts, or variation in the definition of PNI or method of analysis.

Several studies have suggested that other measures of PNI may be more helpful than simply reporting its presence or absence. Of these, the only variables that were independently associated with progression on multivariable analysis were number of extraprostatic nerves infiltrated $(\geq 10)^{2}$ and PNI diameter. ${ }^{11}$

Other research has investigated $\mathrm{PNI}$ on prostate biopsy as a predictor of extraprostatic extension (EPE) at RP. A recent meta-analysis of 10 studies reported that a significantly higher incidence of EPE was seen in patients with PNI at needle biopsy. ${ }^{17}$ However, the authors acknowledge that the main limitation of their analysis was that it was not possible to perform a multivariable analysis. As such, they cannot conclude the presence of $\mathrm{PNI}$ on prostate biopsy alone can guide the urologist in clinical workup.

\section{Conclusion}

Routine reporting of presence or absence of PNI should be questioned, as we have no evidence that it directly informs clinical practice. Our results do not address the potential utility of more nuanced measures of PNI, such as PNI diameter or number of nerves infiltrated, to inform clinical decision-making. Although the pathological process of PNI may be significant to the progression of prostate cancer, further research is needed to understand its biology before clinical studies can be designed that utilize this information in a clinically meaningful way.

Acknowledgments: The authors would like to thank Dr. John Pedersen for his advice.
Competing interests: The authors declare no competing financial or personal interests.

This paper has been peer-reviewed.

\section{References}

1. Ayala GE, Dai H, Ittmann $M$, et al. Growth and survival mechanisms associated with perineural invasion in prostate cancer. Cancer Res 2004;64:6082-90. http://dx.doi.org/10.1158/0008-5472.CAN-04-0838

2. Aumayr K, Breitegger M, Mazal PR, et al. Quantification of extraprostatic perineural spread and its prognostic value in pT3a pNO MO RO prostate cancer patients. Prostate 2011;71:1790-5. http:// dx.doi.org/10.1002/pros.21396

3. Merrilees $\mathrm{AD}$, Bethwaite $\mathrm{PB}$, Russell $\mathrm{GL}$, et al. Parameters of perineural invasion in radical prostatectomy specimens lack prognostic significance. Mod Pathol 2008;21:1095-100. http://dx.doi.org/10.1038/ modpathol.2008.81

4. Marchesi F, Piemonti L, Mantovani A, et al. Molecular mechanisms of perineural invasion, a forgotten pathway of dissemination and metastasis. Cytokine and Growth Factor Reviews 2010;21:77-82. http:// dx.doi.org/10.1016/i.cytogfr.2009.11.001

5. Liebig C, Ayala G, Wilks JA, et al. Perineural invasion in cancer. Cancer 2009;115:3379-91. http:// dx.doi.org/10.1002/cncr.24396

6. Ayala GE. Stromal antiapoptotic paracrine loop in perineural invasion of prostatic carcinoma. Cancer Res 2006;66:5159-64. http://dx.doi.org/10.1158/0008-5472.CAN-05-1847

7. Endrizzi J, Seay T. The relationship between early biochemical failure and perineural invasion in pathological T2 prostate cancer. BJU Int 2000;85:696-8. http://dx.doi.org/10.1046/i.1464-410x.2000.00518.x

8. Jeon $\mathrm{HG}, \mathrm{Bae} J$, $Y \mathrm{i} J \mathrm{~S}$, et al. Perineural invasion is a prognostic factor for biochemical failure after radical prostatectomy. Int J Urol 2009;16:682-6. http://dx.doi.org/10.1111/i.1442-2042.2009.02331.x

9. Jung JH, Lee JW, Arkoncel FRP, et al. Significance of perineural invasion, lymphovascular invasion, and high-grade prostatic intraepithelial neoplasia in robot-assisted laparoscopic radical prostatectomy. Ann Surg Oncol 2011;18:3828-32. http://dx.doi.org/10.1245/s10434-011-1790-4

10. Lee JT, Lee $S$, Yun CJ, et al. Prediction of perineural invasion and its prognostic value in patients with prostate cancer. Korean J Urol 2010;51:745. http://dx.doi.org/10.4111/kju.2010.51.11.745

11. Maru N, Ohori M, Kattan MW, et al. Prognostic significance of the diameter of perineural invasion in radical prostatectomy specimens. Hum Pathol 2001;32:828-33. http://dx.doi.org/10.1053/ hupa.2001.26456

12. Miyake H, Sakai I, Harada KI, et al. Limited value of perineural invasion in radical prostatectomy specimens as a predictor of biochemical recurrence in Japanese men with clinically localized prostate cancer. Hinyokika Kiyo 2005;51:241-6.

13. Ozcan F. Correlation of perineural invasion on radical prostatectomy specimens with other pathologic prognostic factors and PSA failure. Eur Urol 2001;40:308-12. http://dx.doi.org/10.1159/000049791

14. Ravery V, Boccon-Gibod LA, Meulemans A, et al. Predictive value of pathological features for progression after radical prostatectomy. Eur Urol 1994;26:197-201.

15. van den Ouden D, Hop WC, Kranse R, et al. Tumour control according to pathological variables in patients treated by radical prostatectomy for clinically localized carcinoma of the prostate. Br J Urol 1997;79:20311. http://dx.doi.org/10.1046/i.1464-410X.1997.33011.x

16. Ramsden AR, Chodak G. An analysis of risk factors for biochemical progression in patients with seminal vesicle invasion: Validation of Kattan's nomogram in a pathological subgroup. BJU Int 2004;93:961-4. http://dx.doi.org/10.1111/i.1464-410X.2003.04760.x

17. Cozzi G, Rocco BM, Grasso A, et al. Perineural invasion as a predictor of extraprostatic extension of prostate cancer: A systematic review and meta-analysis. Scand J Urol 2013;47:1-6. http://dx.doi.org $/ 10.3109 / 21681805.2013 .776106$

Correspondence: Dr. Fairleigh Reeves, Department of Urology, The Royal Melbourne Hospital, Level 3 Centre, Grattan St., Parkville, Victoria, Australia 3050; fairleighr@gmail.com 